\title{
Circuit Breaker Shunt Resistance Effect on Ferroresonance in Voltage Transformer Including Nonlinear Core Losses
}

\author{
Hamid Radmanesh, Matin Nademi, and Maryam Nademi
}

\begin{abstract}
Ferroresonance is a nonlinear resonance, which may cause overvoltages in the electrical power system. This paper studies the effect of circuit breaker shunt resistance on the control of ferroresonance in a voltage transformer while the core losses of the VT core are highly nonlinear. It is expected that this resistance generally can cause ferroresonance 'dropout'. For confirmation this aspect simulation has been done on a one phase voltage transformer rated 100VA, $275 \mathrm{kV}$. The magnetization characteristic of the transformer is modeled by a single-value two-term polynomial with $q=7$. The simulation results reveal that considering the shunt resistance on the circuit breaker in the case of nonlinear core losses, exhibits a great mitigating effect on ferroresonance overvoltages. Significant effect on the onset of chaos, the range of parameter values that may lead to chaos along with ferroresonance overvoltages has been obtained and presented.
\end{abstract}

Index Terms-Circuit breakers shunt resistance, nonlinear core losses effect, chaos, bifurcation, ferroresonance, voltage transformers

\section{INTRODUCTION}

Ferroresonance overvoltage on electrical power systems were recognized and studied as early as 1930s. Kieny first suggested applying chaos to the study of ferroresonance in electric power circuits [1]. He studied the possibility of ferroresonance in power system, particularly in the presence of long capacitive lines as highlighted by occurrences in France in 1982, and produced a bifurcation diagram indicating stable and unstable areas of operation. Then the combination of nonlinear iron core inductor with series capacitor has been investigated and shown that this core is the most possible case for occurring ferroresonance in the power system. These capacitances can be due to number of elements, such as the line-to-line capacitance, parallel lines, conductor to earth capacitance and circuit breaker grading capacitance. Special ferroresonance phenomena on 3-phase $66 \mathrm{kV}$ VT-generation of $20 \mathrm{~Hz}$ zero sequence continuous voltage is given in [2]. Typical cases of ferroresonance are reported in [3], [4], in these papers power transformer and VTs has been investigated due to ferroresonance overvoltages. Digital simulation of transient in power system has been done in [5]. Application of nonlinear dynamics and chaos to ferroresonance in the distribution systems can be found in [6]. The susceptibility of a ferroresonance circuit to

Manuscript received September 9, 2012; revised October 23, 2012.

Hamid Radmanesh is with Electrical Engineering Department, Shahab Danesh Institute of Higher Education, ghom, Iran(e-mail: Hamid.radmanesh@aut.ac.ir).

Matin Nademi and M. Nademi are with Department of Electrical Engineering, Islamic Azad University, Takestan Branch, Takestan, Iran(e-mail: hrbscstudent@gmail.com; mam.nademi@gmail.com). a quasi periodic and frequency locked oscillations has been presented in [7], in this case, investigation of ferroresonance has been done upon the new branch of chaos theory that is quasi periodic oscillation in the power system and finally ferroresonance appears by this route. Modeling iron core nonlinearities has been illustrated in [8]. Mozaffari has been investigated the ferroresonance in power transformer and effect of initial condition on this phenomena, he analyzed condition of occurring chaos in the transformer and suggested the reduced equivalent circuit for power system including power switch and trans [9],[10]. The mitigating effect of transformer connected in parallel to a MOV arrester has been illustrated in [11]. Analysis of ferroresonance in voltage transformer has been investigated by Zahawi in [12] and [13]. Analysis of ferroresonance phenomena in the power transformers including neutral resistance effect has been reported in [14]. Ferroresonance conditions associated with a $13 \mathrm{kV}$ voltage regulator during back-feed conditions are given in [15]. Performance of various magnetic core models in comparison with the laboratory test results of a ferroresonance test on a $33 \mathrm{kV}$ voltage transformer investigated in [16]. Mitigating ferroresonance in voltage transformers in ungrounded MV networks has been reported in [17]. An approach for determining the subsystem experiencing and producing a bifurcation in a power system dynamic model has been reported in [18]. In all previous studies, possibility of occurring ferroresonance and nonlinear phenomena in power system had been studied and control of this unwanted phenomena has not been studied, also the effects of circuit breaker shunt resistance on VT ferroresonance in the deeper case has not been investigated. Current paper studies the effect of circuit breaker shunt resistance on the control of ferroresonance overvoltages in VT including nonlinear core losses effect. It is shown that by considering this resistance, the behavior of system has been changed and ferroresonance drop out. By modeling the core losses with nonlinear model, it has been shown the nonlinear core losses can cause more chaotic overvoltages when it compares with the linear core model.

\section{System Description Without CB Shunt Resistance}

During VT ferroresonance an oscillation occurs between the nonlinear iron core inductance of the VT and existing capacitances of network. In this case, energy is coupled to the nonlinear core of the voltage transformer via the open circuit breaker grading capacitance or system capacitance to sustain the resonance. The result may be saturation in the VT core and very high voltage up to $4 \mathrm{pu}$ can theoretically gained in worst case conditions. The magnetizing characteristic of a typical 100VA VTs can be presented by 7 order polynomial 
[12]. Fig. 1 shows the single line diagram of the most commonly encountered system arrangement that can give rise to VT ferroresonance [13]. Ferroresonance can occur upon opening of disconnector 3 with circuit breaker open and either disconnector 1 or 2 closed. Alternatively it can also occur upon closure of both disconnector 1 or 2 with circuit breaker and disconnector 3 open.

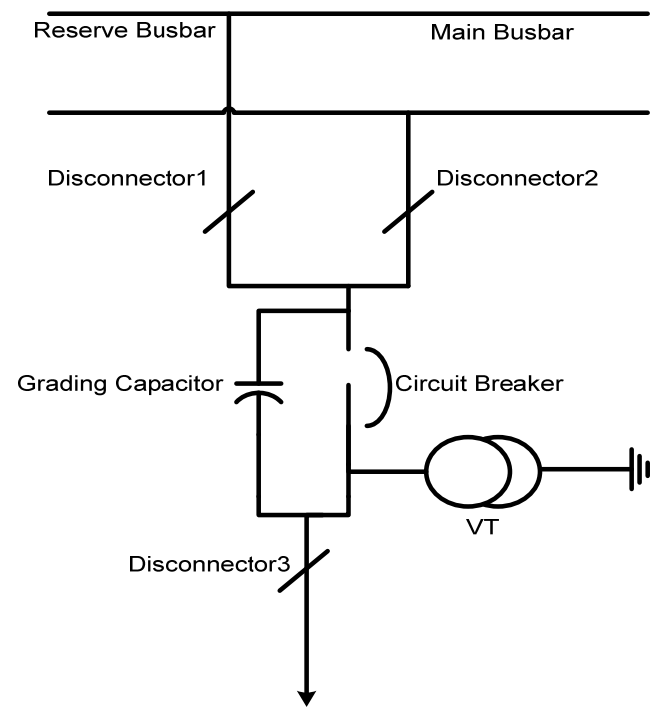

Fig. 1. System one line diagram arrangement resulting to VT ferroresonance

The system arrangement shown in Fig. 1 can effectively be reduced to an equivalent circuit as shown in Fig. 2.

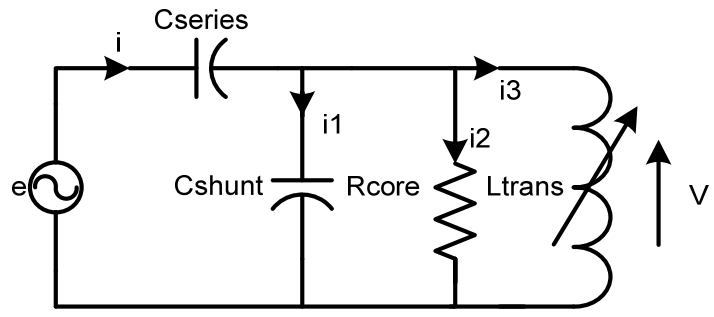

Fig. 2. Basic reduced equivalent ferroresonance circuit [13]

In Fig. 2, $E$ is the RMS supply phase voltage, $C_{\text {series }}$ is the circuit breaker grading capacitance and $C_{\text {shunt }}$ is the total phase-to-earth capacitance of the arrangement. The resistor $R$ represents a voltage transformer core loss that has been found to be an important factor in the initiation of ferroresonance. In the peak current range for steady-state operation, the flux-current linkage can be approximated by a linear characteristic such as $i_{L}=a \lambda$ where the coefficient of the linear term (a) corresponds closely to the reciprocal of the inductance $(a \cong 1 / L)$. However, for very high currents the iron core might be driven into saturation and the flux-current characteristic becomes highly nonlinear, here the $\lambda-i$ characteristic of the voltage transformer is modeled as in [8] by the polynomial

$$
i=a \lambda+b \lambda^{7}
$$

where, $a=3.14, b=0.41$

The Fig. 3 shows simulation of these iron core characteristic for $\mathrm{q}=5,7,11$. The basic voltage transformer ferroresonance circuit of Fig. 2 can be presented by a differential equation. Because of the nonlinear nature of the transformer magnetizing characteristics, the behaviour of the system is extremely sensitive to change in system parameter and initial conditions. A small change in the value of system voltage, capacitance or losses may lead to dramatic change in the behaviour of it. A more suitable mathematical language for studying ferroresonance and other nonlinear systems is provided by nonlinear dynamic methods. Mathematical tools that are used in this analysis are phase plan diagram, time domain simulation and bifurcation diagram.

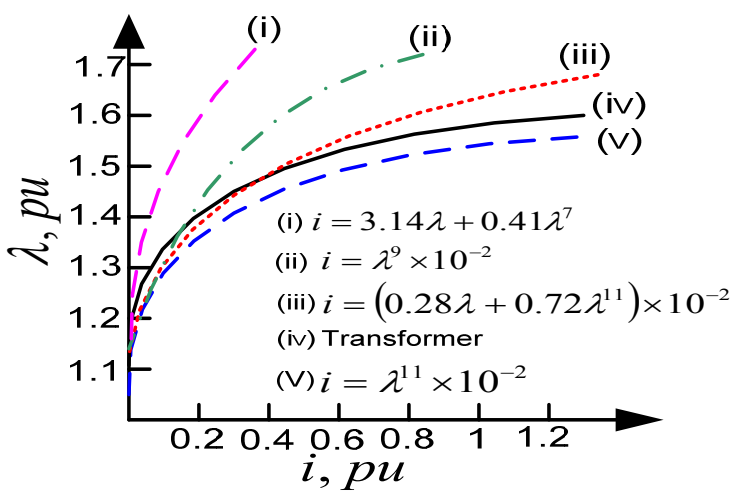

Fig. 3. Nonlinear characteristics of transformer core with different values of $q$

\section{SYSTEM DYNAMIC AND EQUATION}

In this paper, the core loss model adopted is described by a third order power series which coefficients are fitted to match the hysteresis and eddy current nonlinear characteristics given in [6]:

$$
i_{R m}=h_{0}+h_{1} V_{L}+h_{2} V_{L}^{2}+h_{3} V_{L}^{3}
$$

Per unit value of $\left(i_{R m}\right)$ is given in (3)

$$
i_{R m}=-0.000001+0.004 W_{L}-0.007 \mathcal{Y}_{L}^{2}+0.003 W_{L}^{3}
$$

Mathematical analysis of equivalent circuit by applying KVL and KCL has been done and equations of system can be presented as below:

$$
\begin{gathered}
\lambda_{\text {peak }}=\sqrt{2} \frac{v_{R M S}}{\omega} \\
v_{L}=\frac{d \lambda}{d t} \\
i=C_{s e r} \frac{d\left(e-v_{L}\right)}{d t}=C_{s e r}\left(\dot{e}-\frac{d^{2} \lambda}{d t^{2}}\right) \\
\frac{C_{\text {series }}}{\left(C_{\text {ser }}+C_{s h}\right)}(\sqrt{2} E \cos \omega t)=\frac{1}{\omega} \frac{d^{2} \lambda}{d t^{2}}+\frac{1}{\omega\left(C_{\text {ser }}+C_{\text {sh }}\right)} \\
\times\left(\mathrm{h}_{0}+\mathrm{h}_{1} \frac{d \lambda}{d t}+\mathrm{h}_{2}\left(\frac{d \lambda}{d t}\right)^{2}+\mathrm{h}_{3}\left(\frac{d \lambda}{d t}\right)^{3}+a \lambda+b \lambda^{7}\right)
\end{gathered}
$$

where, $\omega$ is supply frequency, and $E$ is the rms supply phase 
voltage, $C_{\text {series }}$ is the circuit breaker grading capacitance and $C_{\text {shunt }}$ is the total phase-to-earth capacitance of the arrangement and in (1) $a=3.4$ and $b=0.41$ are the seven order polynomial sufficient [13].

\section{Metal System Description with CB Shunt RESISTANCE}

In this case, system under study is similar with the case above, but the model of circuit breaker has been changed. Equivalent circuit of this case has been illustrated in Fig. 4.

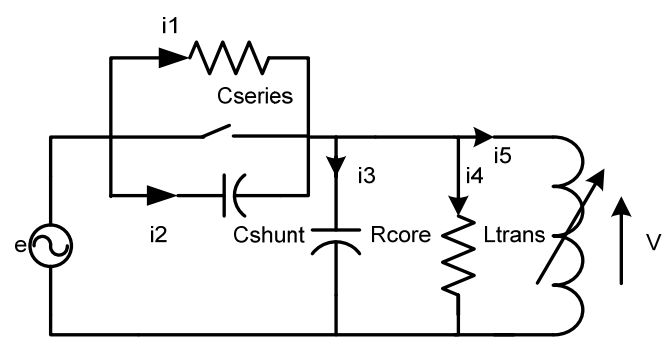

Fig. 4. Basic reduced equivalent ferroresonance circuit

Nonlinear Equation of this circuit is given in (8).

$$
\begin{aligned}
& \frac{1}{R_{C . B}\left(C_{s e r}+C_{s h}\right)} \cdot\left(\sqrt{2} E \operatorname{Sin}(\omega t)-\frac{d \lambda}{d t}\right)+\frac{C_{s e r}}{\left(C_{s e r}+C_{s h}\right)} \\
& \times \sqrt{2} \omega E \operatorname{Cos}(\omega t)=\frac{d^{2} \lambda}{d t^{2}}+\frac{1}{\left(C_{s e r}+C_{s h}\right)}\left(\left(a \lambda+b \lambda^{7}\right)+\right. \\
& \left(h_{0}+h_{1} v_{L}+h_{2} v_{L}^{2}+h_{3} v_{L}^{3}\right)
\end{aligned}
$$

In this model of circuit, $\mathrm{R}_{\mathrm{CB}}$ is paralleled with the $\mathrm{C}_{\text {series }}$ and its value is $R_{C B}=0.34 \mathrm{pu}$. Other parameters of system are similar with the case 1 .

\section{Simulation Results}

TABLE I: PARAMETERS VALUE FOR SIMULATION

\begin{tabular}{|l|l|l|}
\hline Parameter & Actual value & Per unit value \\
\hline$E$ & $275 \mathrm{kv}$ & $1 \mathrm{pu}$ \\
\hline$\omega$ & $377 \mathrm{rad} / \mathrm{sec}$ & $1 \mathrm{pu}$ \\
\hline$C_{\text {series }}$ & $0.5 \mathrm{nf}$ & $39.959 \mathrm{pu}$ \\
\hline$C_{\text {shunt }}$ & $0.1 \mathrm{nf}$ & $7.92 \mathrm{pu}$ \\
\hline Rcore & $225 \mathrm{M} \Omega$ & $0.89 \mathrm{pu}$ \\
\hline
\end{tabular}

Values of $E$ and $\omega$ were fixed at 1pu, corresponding to $\mathrm{AC}$ supply voltage and frequency. $C_{\text {series }}$ is the CB grading capacitance and its value obviously depends on the type of circuit breaker. In this analysis $C_{\text {series }}$ is fixed at $0.5 \mathrm{nF}$ and $C_{\text {shunt }}$ vary between $0.1 \mathrm{nF}$ and $3 \mathrm{nF}$.solutions are obtained for initial values of $V(t)=\sqrt{2}, \lambda(t)=0$ at $\mathrm{t}=0$, representing circuit breaker operation at maximum voltage. In this state, system for both cases, with and without circuit breaker shunt resistance has been simulated for $E=3 \mathrm{pu}$. it shows that the system under study has a chaotic behaviour for $E=3 p u$. Corresponding phase plan diagrams has been shown the clearance effect of applying the shunt resistance to the system and it is shown in Figs. 7 and 8 for $E=3$ pu. System parameters are listed in Table I.

Fig 5, 6 show time domain simulation for $E=3 p u$ in the case of considering shunt resistance it has been shown the effect of shunt resistance on system behavior.

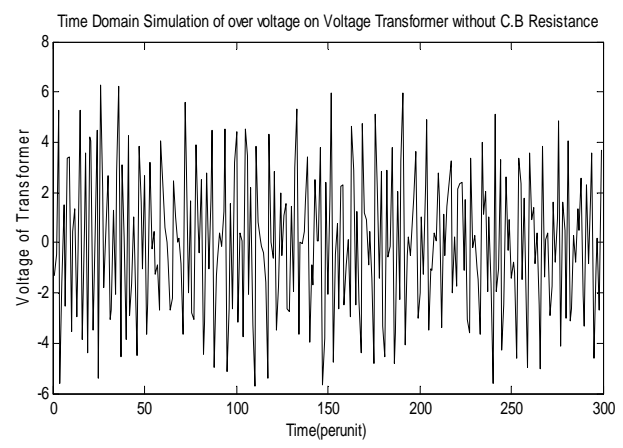

Fig. 5. Time domain simulation for chaotic motion without $\mathrm{CB}$ shunt resistance, $E=4 p u$

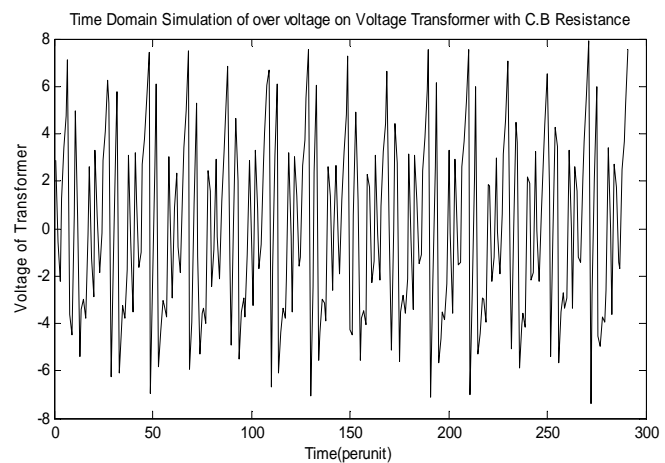

Fig. 6. Time domain simulation for clamping the chaotic motion considering CB shunt resistance effect, $E=4 p u$

In Fig. 5 System behavior has been simulated without considering shunt resistance. Time domain simulation is completely chaotic and ferroresonance overvoltage reaches to $6 \mathrm{pu}$. In the equal condition, by applying shunt resistance, this overvoltage has been damped and behavior of system goes to linear region. According to the Fig. 6, system frequency is equal the periodic condition and voltage of transformer has been fixed to $1.8 \mathrm{pu}$. In the case of without considering shunt resistance effect, ferroresonance overvoltage on voltage transformer reaches to $6 \mathrm{pu}$, and state has been shown by phase plan diagram in Fig. 7.

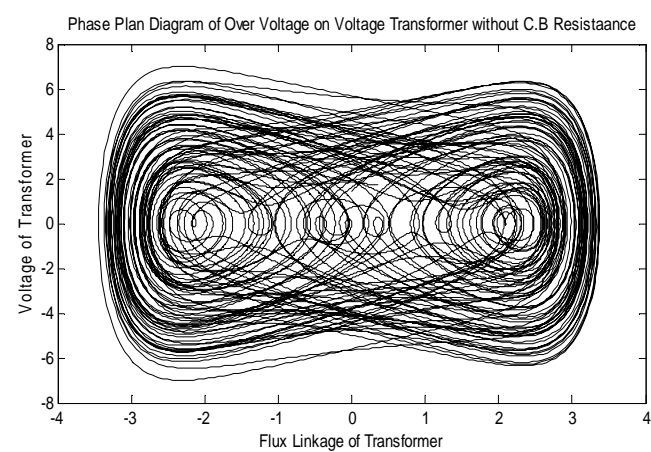

Fig. 7. Phase plan diagram for chaotic motion without $\mathrm{CB}$ shunt resistance, $\mathrm{E}=4 \mathrm{pu}$

By applying shunt resistance effect to the system while the input voltage is $4 \mathrm{pu}$, chaotic oscillation is changed to the fundamental resonance as shown in Fig. 8 that ferroresonance overvoltages clamp to $1.6 \mathrm{pu}$ and it is presented in Fig. 8. 


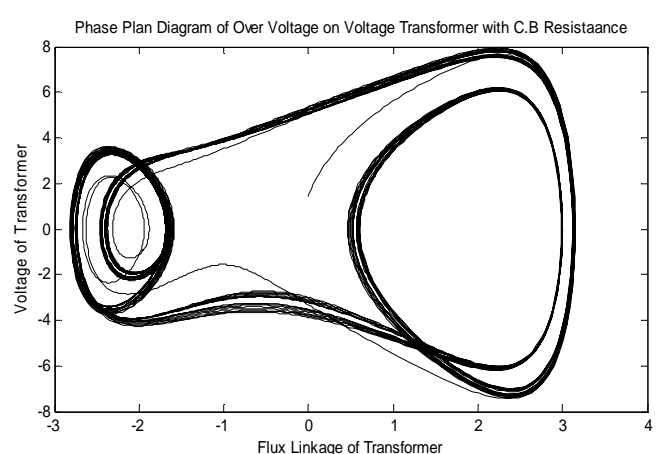

Fig. 8. Phase plan diagram for clamping the chaotic motion with $\mathrm{CB}$ shunt resistance, $\mathrm{E}=4 \mathrm{pu}$

It obviously shows that circuit breaker shunt resistance clamps the ferroresonance overvoltage and keeps it in $\mathrm{E}=2 \mathrm{pu}$. System parameters which are considered for these cases of simulation are listed in Table II.

\begin{tabular}{|l|l|l|}
\hline \multicolumn{1}{|c|}{ TABLE II: PARAMETER VALUE FOR SIMULATION } \\
\hline Parameter & Actual value & $\begin{array}{l}\text { Per unit } \\
\text { value }\end{array}$ \\
\hline$E$ & $275 \mathrm{kv}$ & $1 \mathrm{pu}$ \\
\hline$\omega$ & $377 \mathrm{rad} / \mathrm{sec}$ & $1 \mathrm{pu}$ \\
\hline$C_{\text {series }}$ & $0.5 \mathrm{nf}$ & $\begin{array}{l}39.959 \\
\mathrm{pu}\end{array}$ \\
\hline$C_{\text {shunt }}$ & $1.25 \mathrm{nf}$ & $99 \mathrm{pu}$ \\
\hline & & \\
\hline
\end{tabular}

In this paper, it is shown the effect of variation in the voltage of system on the ferroresonance overvoltage in the VT, and finally the effect of applying circuit breaker shunt resistance on this overvoltage by the bifurcation diagrams.

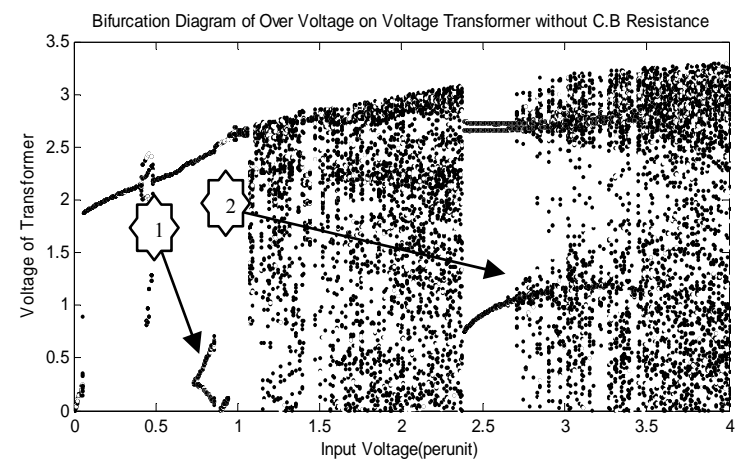

Fig. 9. Bifurcation diagram for voltage of transformer versus voltage of system, without $\mathrm{CB}$ shunt resistance effect

Fig. 9 clearly shows the ferroresonance overvoltages on VT when the voltage of system increases to 4 pu. Parameters value of the system in this case is listed in Table III.

TABLE III: PARAMETER VALUE FOR SIMULATION
\begin{tabular}{|l|l|l|}
\hline Parameter & Actual value & $\begin{array}{l}\text { Per unit } \\
\text { value }\end{array}$ \\
\hline$E$ & $275 \mathrm{kV}$ & $1 \mathrm{pu}$ \\
\hline$w$ & $377 \mathrm{rad} / \mathrm{sec}$ & $1 \mathrm{pu}$ \\
\hline$C_{\text {series }}$ & $0.5 \mathrm{nf}$ & $39.959 \mathrm{pu}$ \\
\hline$C_{\text {shunt }}$ & $0.1 \mathrm{nf}$ & $7.92 \mathrm{pu}$ \\
\hline & & \\
\hline
\end{tabular}

In Fig. 9 when $\mathrm{E}=0.25 \mathrm{pu}$, voltage of VT has a period- 1 behavior and system works under normal condition, in $\mathrm{E}=0.57 \mathrm{pu}$ that has been shown by point -1 , its behavior is period-3 and after this voltage, suddenly crisis takes place and system behavior goes to the chaotic region. After that, when the input voltage reach to $2.2 \mathrm{pu}$, system comes out of chaotic region, again in the point 2 and 3, bifurcation takes place. By this route system behavior goes to chaos. It is shown that system behavior has period doubling bifurcation logic and there are many resonances in the system behavior. Bifurcation diagram with the same parameter in the case of applying CB shunt resistance parallel to the VT is shown in Fig. 10.

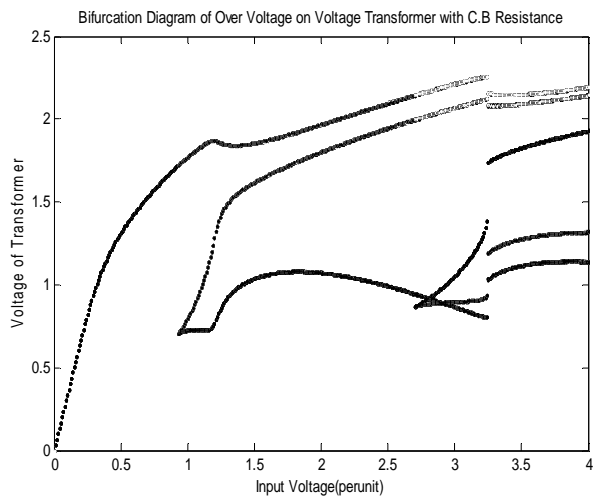

Fig. 10. Bifurcation diagram for voltage of transformer versus voltage of system considering CB shunt resistance effect

It is shown that by applying this resistance, system behaviors coming out of chaotic region and $\mathrm{CB}$ shunt resistance can clamp overvoltages. In the real systems, maximum overvoltage that VT can stand is $4 \mathrm{pu}$. By comparing Figs. 9, and 10, it has been shown that the nonlinear core losses have a big effect of occurring ferroresonance. If overvoltage increases more, it can exactly cause VT failure.

\section{CONCLUSION}

Low capacity VTs fed through circuit breaker grading capacitance have been shown to exhibit fundamental frequency and chaotic ferroresonance conditions similar to high capacity power transformers fed via capacitive coupling from neighbouring sources. Repeated simulation of the system's nonlinear differential equation has shown that a change in the value of the equivalent circuit capacitance to earth, possibly as a result of a change in system configuration, can give rise to different types of ferroresonance overvoltage. CB shunt resistance successfully can cause ferroresonance drop out and can control it. A comprehensive understanding of the possibilities that exist for ferroresonance is very desirable for engineers so that they can operate their systems outside dangerous regions and can plan the expansion of systems without enhancing the possibility of ferroresonance.

\section{ACKNOWLEDGMENT}

Corresponding author would like to appriciate Dr. Ali Nasrabadi of the Shahed University, Tehran, Iran, for providing MATLAB data files for the time domain simulations, and Mrs. Leila Kharazmi for her English editing.

\section{REFERENCES}

[1] Kieny, "Application of the bifurcation theory in studying and understanding the global behavior of a ferroresonant electric power 
circuit," IEEE Transactions on Power Delivery, vol. 6, 1991, pp. 866-872.

[2] S. Nishiwaki, T. Nakamura, and Y .Miyazaki, "A Special Ferro-resonance Phenomena on 3-phase 66kV VT-generation of $20 \mathrm{~Hz}$ zero sequence continuous voltage," Presented at the International Conference on Power Systems Transients (IPST'07), in Lyon, France on June 4-7, 2007.

[3] E. J. Dolan, D. A. Gillies, and E. W. Kimbark, "Ferroresonance in a transformer switched with an EVH line," IEEE Transactions on Power Apparatus and Systems, vol, 1972, pp. 1273-1280.

[4] R. P. Aggarwal, M. S. Saxena, B. S. Sharma, S. Kumer, and S. Krishan, "Failure of electromagnetic voltage transformer due to sustained overvoltage on switching*/an in-depth field investigation and analytical study," IEEE Transactions on Power Apparatus and Systems, vol. 5, 1981, pp. 4448-4455.

[5] E. P. Dick and W. Watson, 'Transformer models for transient studies based on field measurements," IEEE Trans, 1981, PAS-100, pp. 409417.

[6] H. W. Dommel, A. Yan, R. J. O. D. Marcano, A. B. Miliani, in: H.P Khincha(Ed.), Tutorial Course on Digital Simulation of Transients in Power Systems (Chapter 14), IISc, Bangalore, 1983, pp. 17-38.

[7] B. A. Mork and D. L. Stuehm, "Application of nonlinear dynamics and chaos to ferroresonance in distribution systems," IEEE Transactions on Power Delivery, vol. 9, 1994, pp. 1009-1017.

[8] S. K. Chkravarthy and C. V. Nayar, "Frequency-locked and quasi periodic (QP) oscillations in power systems," IEEE Transactions on Power Delivery, vol. 13, 1997, pp. 560-569.

[9] W. L. A. Neves and H. Dommel, "on modeling iron core nonlinearities," IEEE Transactions on Power Systems, vol. 8, 1993, pp. $417-425$.

[10] S. Mozaffari, M. Sameti, and A. C. Soudack, "Effect of initial conditions on chaotic ferroresonance in power transformers, IEE Proceedings*/Generation," Transmission and Distribution, vol. 144, 1997, pp. 456-460.

[11] S. Mozaffari, S. Henschel, and A. C. Soudack, "Chaotic ferroresonance in power transformers," in Proc. IEE Generation, Transmission Distrib, vol. 142, 1995, pp. 247-250.

[12] K. A. Anbarri, R. Ramanujam, T. Keerthiga, and K. Kuppusamy, "Analysis of nonlinear phenomena in MOV connected Transformers," IEE Proceedings */Generation Transmission and Distribution1, vol.48, 2001, pp. 562-566.
[13] B.A.T. Al Zahawi, Z. Emin, Y.K. Tong, Chaos in ferroresonant wound voltage transformers: effect of core losses and universal circuit behavioral, IEE Proceedings*/Sci. Measurement Technology, vol. 145, 1998, pp. 39-43.

[14] Z. Emin, B. A. T. A. Zahawi, D. W. Auckland, and Y. K. Tong, "Ferroresonance in Electromagnetic Voltage Transformers: A Study Based on Nonlinear Dynamics," in IEE Proc. on Generation, Transmission, Distribution, vol. 144, 1997, pp. 383-387.

[15] H. Radmanesh, A. Abassi, and M. Rostami, "Analysis of ferroresonance phenomena in power transformers including neutral resistance effect," Southeastcon, 2009. SOUTHEASTCON '09. IEEE, pp. 1-5, 5-8 March 2009.

[16] D. Shoup, J. Paserba, and A. Mannarino, "Ferroresonance Conditions Associated with a $13 \mathrm{kV}$ Voltage Regulator During Back-feed Conditions," Presented at the International Conference on Power Systems Transients (IPST'07), vol. 2, 2007, pp.1212-1215.

[17] A. R. Zare, H. Mohseni, M. S. Pasand, S. Farhangi, and R. Iravani, "Performance of Various Magnetic Core Models in Comparison with the Laboratory Test Results of a Ferroresonance Test on a $33 \mathrm{kV}$ Voltage Transformer," Presented at the International Conference on Power Systems Transients (IPST'07), in Lyon, France on June 4-7, 2007.

[18] W. Piasecki, M. Florkowski, M. Fulczyk, P. Mahonen, and W. Nowak, "Mitigating Ferroresonance in Voltage Transformers in Ungrounded MV Networks," IEEE Transaction on power delivery, vol. 22, no. 4, 2007.

[19] K. B. Kilani and R. A. Schlueter, "An Approach for Determining the Subsystem Experiencing and Producing a Bifurcation in a Power System Dynamic Model," IEEE Transaction on power systems, vol. 15 , no. 3, 2000, pp. 1053-1061 .

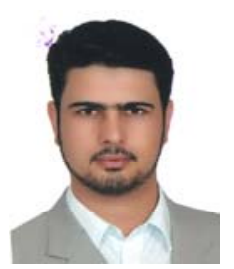

Hamid Radmanesh was born in 1981. He studied Telecommunication engineering at Malek-Ashtar University of Technology, Tehran, Iran, and received the BSC degree in 2006, also studied electrical engineering at Shahed University Tehran, Iran, and received the MSC degree in 2009. Currently, $\mathrm{He}$ is $\mathrm{PhD}$ student in Amirkabir University of Technology. His research interests include design and modeling of power electronic converters, drives, transient and chaos in power system apparatus. 